\title{
Carbonyl Charge Solvation Patterns May Relate to Fragmentation Classes in Collision-Activated Dissociation
}

\author{
Hongqian Yang, ${ }^{1}$ David M. Good, ${ }^{1}$ David van der Spoel, ${ }^{2}$ Roman A. Zubarev ${ }^{1,3}$ \\ ${ }^{1}$ Division of Physiological Chemistry I, Department of Medical Biochemistry and Biophysics, Karolinska Institutet, \\ Stockholm, Sweden \\ ${ }^{2}$ Molecular Biophysics Group, Department of Cell and Molecular Biology, Uppsala University, Uppsala, Sweden \\ ${ }^{3}$ Science for Life Laboratory, Stockholm, Sweden
}

\begin{abstract}
Here, we investigate the hypothesis that the origin of Class I fragmentation in tryptic peptide dications corresponding to the cleavage of the first two amino acids from the $\mathrm{N}$-terminus is due to a dominant charge solvation pattern. Molecular dynamics simulations (MDS) of model $A_{n} R$ dications confirmed the existence of a persistent solvation of the protonated $\mathrm{N}$-terminus on the second backbone carbonyl. Additionally, MDS predicted a new distinct fragmentation class corresponding to the loss of two amino acids from the C-terminus. This prediction was confirmed experimentally at very low excitation levels. The pattern produced by electron transfer dissociation of the same dications gave markedly decreased cleavage frequencies at the second peptide bond, which, within the non-local fragmentation mechanism, supports the preferential charge solvation on the second carbonyl. Taken together, these results confirm the role of a charge solvation pattern in the origin of fragmentation classes.
\end{abstract}

Key words: Peptide fragmentation, Gas-phase chemistry, Sequencing, Protonation

\section{Introduction}

$\mathrm{F}$ ragmentation of peptide polycations is an important step in today's shotgun proteomics. Peptide bond cleavage in activated peptide dications leads to formation of sequence-informative $\mathrm{b}_{n}{ }^{+} / \mathrm{y}_{l-n}{ }^{+}$ion pairs ( $l$ is the peptide length in residues). Understanding the mechanism of peptide bond cleavage is key for improving the efficiency and reliability of peptide sequencing. After more than two decades of studying the fragmentation mechanism in collision-activated dissociation (CAD) [1], several important issues still remain unresolved. A prominent example is the origin of two statistical groups in the fragmentation patterns of tryptic peptide dications [2]. Predominant cleavage after the second N-terminal residue yielding $\mathrm{a} \mathrm{b}_{2}{ }^{+} / \mathrm{y}_{l-2}{ }^{+}$ion pair, the most frequent outcome for tryptic peptide dications is

Correspondence to: Roman Zubarev; e-mail: Roman.Zubarev@ki.se known as Class I behavior. A broad cleavage frequency distribution peaking at the fourth or fifth peptide bond is referred to as Class II fragmentation [2]. The knowledge of different fragmentation classes is important for creating algorithms predicting fragment abundances. But what is the reason for the emergence of the two classes? This question still awaits a definite answer.

Among the first who pointed out that the mechanism of the amide bond cleavage after the second $\mathrm{N}$-terminal residue might differ significantly from that of other peptide bonds were Paizs and Suhai in their highly cited 2005 review [3]. A significant fraction of that work is devoted to detailed description of the "diketopiperazine pathway," the term introduced by the same authors in 2001 [4]. In that pathway, the loss of two neutral amino acids from the N-terminus was initiated by protoninduced trans-to-cis isomerization of the N-terminal amide bond, a process found to be energetically quite feasible but likely kinetically controlled [4]. Such isomerization facilitates the formation of a cyclic-symmetric diketopiperazine structure 
suggested for neutral $b_{2}$ species as early as 1993 [5]. The authors avoided calculating the rate of diketopiperazine formation, admitting that "the quality of the applied theoretical model was not good enough for quantitative predictions" [3].

In 2008, Savitski et al. demonstrated for the first time the dominance of $b_{2} / y_{l-2}$ fragmentation by statistical analysis of $>15,000$ high-resolution mass spectra of tryptic peptide dications [2]. Continuing the line of reasoning of previous investigators, they suggested a different structure of $b_{2}$ ions compared with larger $b$ ions. Since the dominant paradigm at the time for b-ion structure was protonated oxazolone [6-8], Savitski et al. have evoked for Class I fragmentation the diketopiperazine pathway, albeit modified for the ionic case [2]. Subsequent studies by several groups revealed cases of $\mathrm{b}_{2}{ }^{+}$ions being preferentially oxazolones [9-11], as well as diketopiperazines [12]. Although diketopiperazine ionic structures are almost universally found to be more thermodynamically stable than oxazolones [9-12], the evidence supporting the diketopiperazine hypothesis of Class I fragmentation is currently mixed at best. It is worthwhile noting that the critics of the diketopiperazine hypothesis have so far refrained from advancing their own explanation for the fragmentation class emergence.

Another explanation (not necessarily alternative to the previous one) advanced by Savitski et al. in 2006 [13] emphasizes the importance of the charge solvation pattern. A peptide's gas-phase secondary structure arising due to noncovalent bonding, where charge solvation is the strongest of interactions, has long been known to affect peptide fragmentation $[14,15]$. Based on statistical evidence, an Nterminal loop structure has been suggested in gas-phase tryptic peptide dications formed by solvation of the protonated $\mathrm{N}$-terminus on backbone carbonyls [13]. It was proposed that the size of this structure is independent upon the peptide length for $l \geq 7$, and predicted that the most frequent solvation site might be the second carbonyl. It has been shown that the frequency of charge solvation strongly correlates with that bond's fragmentation frequency in CAD [16]. Therefore, frequent charge solvation on the second carbonyl could statistically explain the dominant fragmentation after the second $\mathrm{N}$-terminal residue.

Within the generally accepted mobile proton model [1719], a proton's release from its primary solvation location initiates its drift along the backbone carbonyls (or, more accurately, the drift of the proton's solvation point). Once the proton becomes mobilized, its original position becomes unimportant for fragmentation. The most statistically probable position of a drifting proton is near the center of the polypeptide chain, with a broad distribution of almost as likely positions around that maximum. Class II fragmentation closely corresponds to this picture [2]. However, at lower excitation levels, when the proton could only move a few steps upon mobilization, the most probable proton position remains at the original charge solvation site, with the density distribution decaying with the distance from it. Thus, if the N-terminal proton is preferentially solvated on the second carbonyl, then at low CAD excitations a fragmentation pattern should emerge resembling that of Class I fragmentation [2].

To test this prediction and, thus, the whole classemergence hypothesis, molecular dynamics simulations (MDS) were performed on dications of model peptides $\mathrm{A}_{\mathrm{n}} \mathrm{R}(n=7-9)$ and compared with MS/MS results. The homogeneous poly-Ala sequence was chosen to avoid the effect of different amino acid residues on fragmentation dynamics. In a study by Harrison [20], non-tryptic $\mathrm{A}_{n} \mathrm{H}$ dications showed strong size dependence, with smaller sequences preferring Class I and larger sequences preferring Class II fragmentation. Recently, Harrison studied the effect of amino acid substitution in dications of the seven-residue tryptic peptide A-A-X-X-A-A-A-R [21]. Another reason for choosing Ala-based sequences was that polyalanine peptides have been extensively studied by MDS, and that the early MDS results indicated the presence of an N-terminal, chargestabilized loop structure [22]. In our MDS, the proton was immobilized on the $\mathrm{N}$-terminus. However, it was mobile in the sense that as a part of the protonated amine it could move from a carbonyl to a carbonyl, just as in the mobile proton model.

\section{Experimental}

$A_{n} R$ peptides were synthesized in-house with a ResPep peptide synthesizer (Intavis Bioanalytical Instruments, Cologne, Germany), and then dissolved in a solution of $50 \%$ methanol and $0.1 \%$ formic acid to a concentration of $1 \mu \mathrm{g} / \mu \mathrm{L}$. The peptides were electrosprayed from a metalized pulled glass capillary (Thermo Fisher, CA, USA). MS/MS experiments were performed on an Orbitrap Velos mass spectrometer (Thermo Fisher, CA, USA). In the CAD regime, fragmentation was performed in the linear ion trap (LTQ) through collisions with helium; in higher energy collisional dissociation (HCD), in the "C-trap," through collisions with nitrogen [23]. Electron-transfer dissociation (ETD) was performed in the LTQ [24, 25]. In all cases, the fragment ions were detected in the high-resolution Orbitrap analyzer at $\mathrm{R}=60,000$.

As in our previous studies [26-28], MDS considered uninterrupted time evolution of polypeptide chains with rotating chemical bonds (no vibration). All peptide bonds were considered in trans- configuration; trans-cis isomerization was not modeled.

Linear peptides $A_{7} R, A_{8} R$, and $A_{9} R$ were generated using PyMOL and imported into the GROMACS simulation suite [29, 30]. The OPLS/AA force field was used for all simulations [31, 32]. Replica exchange molecular dynamics (REMD) simulations [33] were performed at eight temperature points $(300,342,388$, 440, 497, 561, 631, $710 \mathrm{~K}$, respectively) which were predicted to lead to an approximate exchange probability of 0.3 [34], leading to an average of $50 \mathrm{ps}$ between exchanges. The simulations were each run six times for $100 \mathrm{~ns}$ with different starting conditions to check for reproducibility. For such small peptides, REMD will 

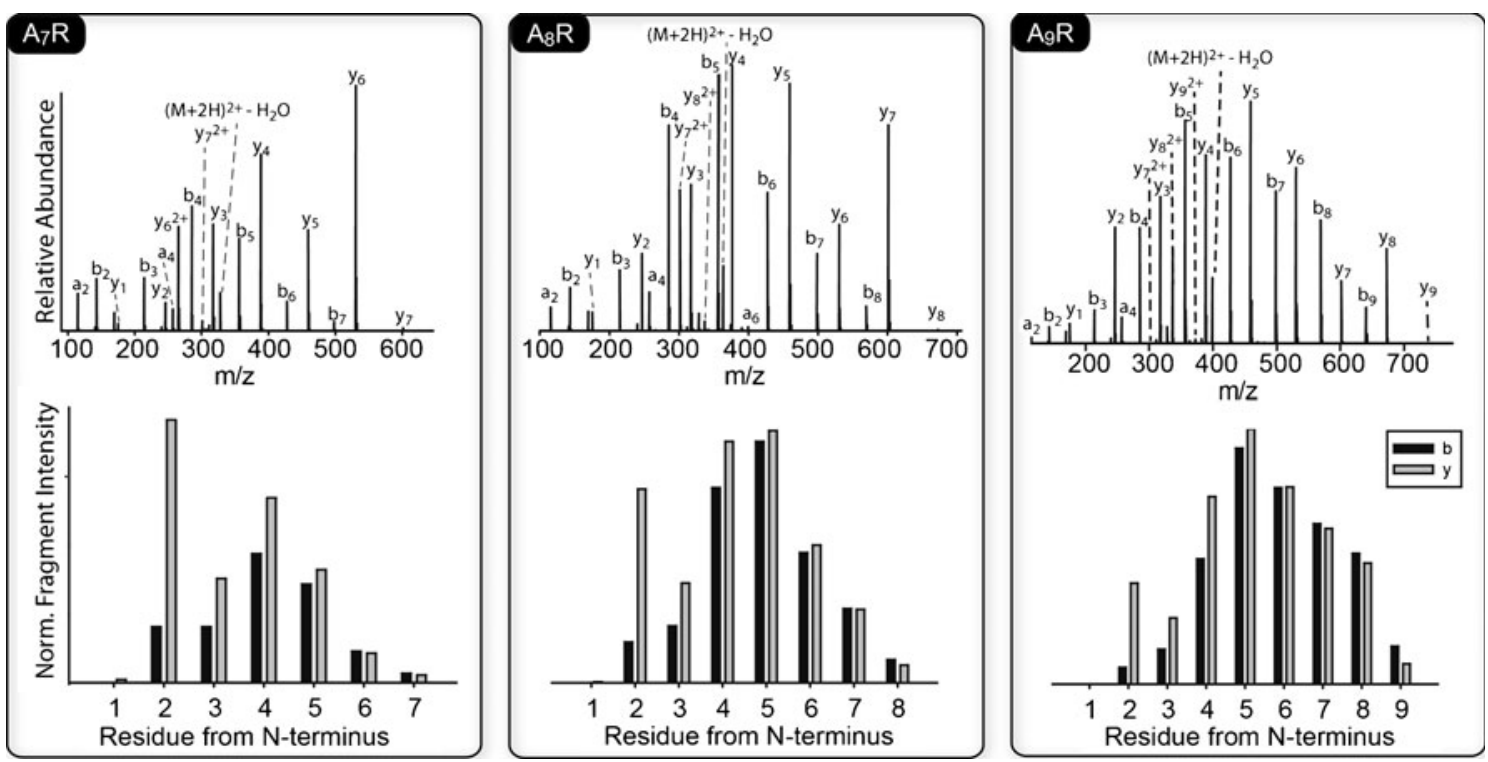

Figure 1. CAD fragmentation $(20 \mathrm{eV} ; 30 \mathrm{~ms})$ of the peptide dications with seven, eight, and nine alanine residues

produce very good conformational sampling in such a time period. A hydrogen bonding analysis was performed to determine the correlation between bonding of the $\mathrm{N}$-terminus and the Arg side chain to the carbonyl oxygens. For this analysis, a geometric criterion of H-bonding was used, where the donor-acceptor distance should be $<0.35 \mathrm{~nm}$, and the hydrogen-donor-acceptor angle $<30^{\circ}$.

The probability maps of charge solvation on every backbone carbonyl were obtained by counting the number of time frames when a charged hydrogen bond existed between the protonated site (either the N-terminus or Arg side chain) and the backbone carbonyls [26].

\section{Results and Discussion}

The results of CAD fragmentation $(20 \mathrm{eV} ; 30 \mathrm{~ms})$ of the peptides with seven, eight, and nine alanine residues are shown in Figure 1. $\mathrm{A}_{7} \mathrm{R}$ showed dominant Class I behavior with $\mathrm{b}_{2} / \mathrm{y}_{l-2}$ fragments having the highest abundance. The two larger molecules gave a mixed mass spectrum, with $\mathrm{A}_{9} \mathrm{R}$ producing a dominant Class II fragmentation pattern, and $\mathrm{A}_{8} \mathrm{R}$, an intermediate result. $\mathrm{HCD}$ at $10 \mathrm{eV}$ (Figure 2) gave a similar fragmentation pattern, with somewhat more pronounced Class I behavior. The new feature in HCD compared withi CAD was the presence of doubly charged $\mathrm{y}$-ions corresponding to preferential losses of two neutral
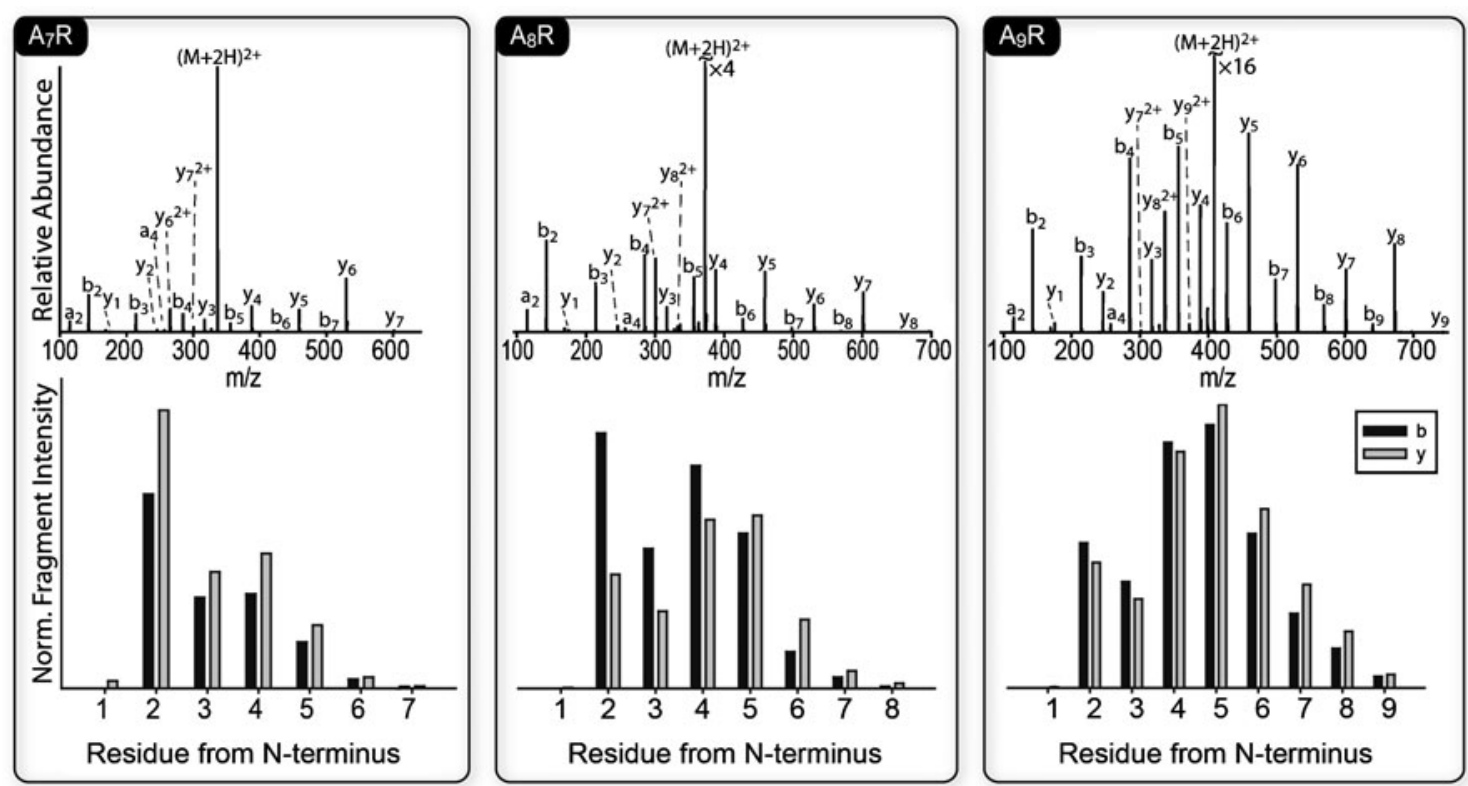

Figure 2. HCD fragmentation of the peptide dications with seven, eight, and nine alanine residues 

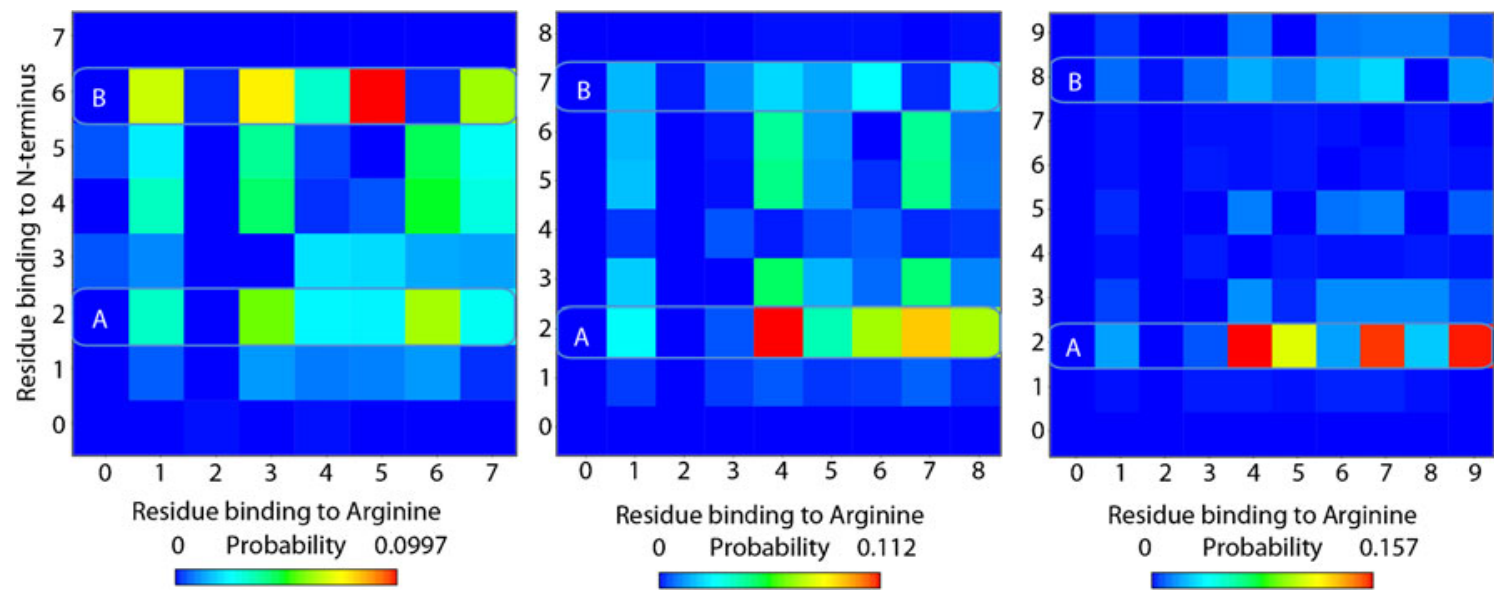

Figure 3. MDS results: relative solvation frequencies of the two protons on backbone carbonyls in tryptic peptide dications of $A_{7} R, A_{8} R$, and $A_{9} R$. Horizontal axis - carbonyl solvating Arg-bound proton; vertical axis - carbonyl solvating $\mathrm{N}$-terminus-bound proton. Zero index corresponds to a nonsolvated state of the protonated group

amino acids from the $\mathrm{N}$-terminus. According to the diketopiperazine pathway by Paizs and Suhai, such neutral losses could occur through formation of neutral diketopiperazine [3]. The formation of a cyclic structure at higher excitation energy is consistent with the recent finding that the sequence scrambling in $\mathrm{b}$ ions [35], which is due to cyclization [36], is more frequent in HCD than in CAD, albeit still occurring with a low probability [37]. Since for sequence scrambling to be detected by mass spectrometry, cyclization of the peptide linear chain must be followed by an additional fragmentation of two peptide bonds, cycle formation per se must be a much more common phenomenon than the detected sequence scrambling.

The CAD and HCD results were compared with the probability maps of charge solvation presented in Figure 3. These maps contain a nearly "empty" diagonal, which is due to the low chance of simultaneous solvation of both protons on the same carbonyl. Zero indices, corresponding to a free (unsolvated) protonated group, are also largely empty, indicating that some kind of charge solvation on backbone carbonyls is almost always present. There is, however, no dominant gas-phase structure in $\mathrm{A}_{\mathrm{n}} \mathrm{R}$ dications, which would be characterized by a well-defined preferred solvation position of both protons. Instead, there seems to be a large collection of inter-converting structures. However, these structures are not totally random; there exist persistent solvation patterns, each pattern representing a distinct area on the solvation density map.

In the high-probability density pattern $\mathrm{A}$, the sites of preferred $\mathrm{N}$-terminal proton solvation are two or, with lower probability, three residues away from the $\mathrm{N}$-terminus regardless of the peptide length. This pattern matches the prediction made [13], and may account for the facile cleavage of the second peptide bond to produce high-
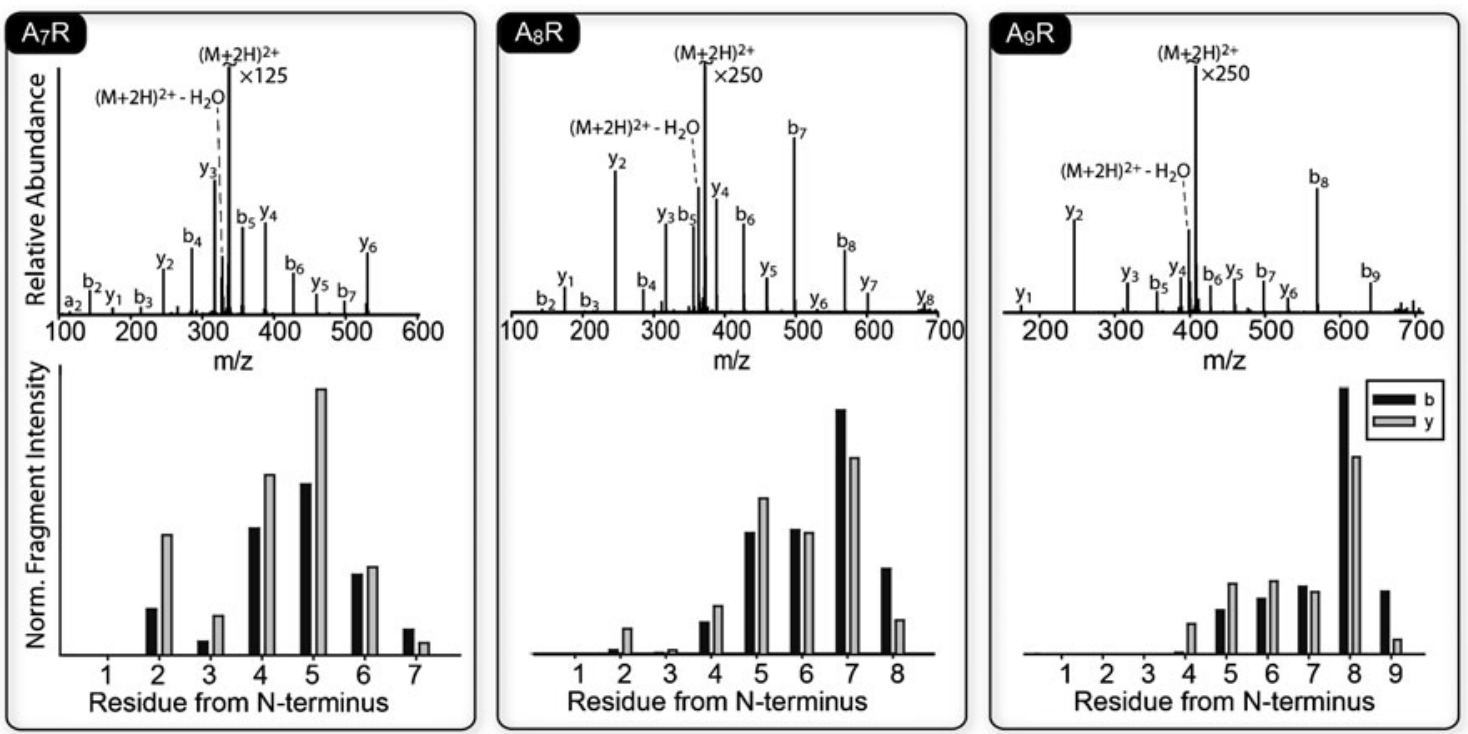

Figure 4. CAD fragmentation $(5 \mathrm{eV} ; 400 \mathrm{~ms})$ of the peptide dications with seven, eight, and nine alanine residues 

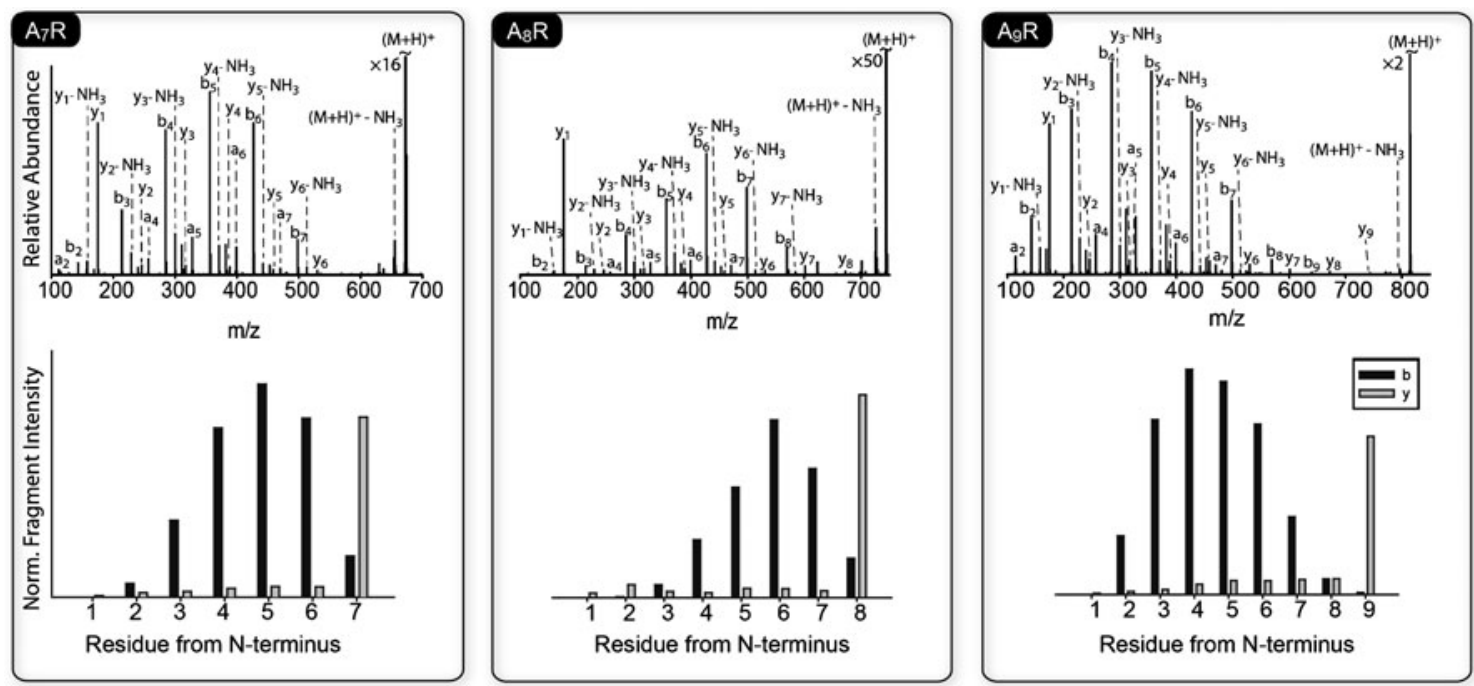

Figure 5. HCD fragmentation of the singly charged peptides with seven, eight, and nine alanine residues

intensity $\mathrm{b}_{2}{ }^{+}$ions. However, the link between the preferred charge solvation on a backbone carbonyl and the probability of cleavage after that carbonyl, although well-established statistically (vide infra), is an indirect one because it does not take into consideration the mechanism of proton transfer from the carbonyl to the amide nitrogen. Such transfer is necessary for the amide bond cleavage because only nitrogen protonation makes the peptide bond weaker, while carbonyl protonation strengthens it [38]. Thus, an additional test of the relevance of the MDS-predicted charge solvation pattern and collisional fragmentation is highly desired. The second region of high probability density in Figure 3 (pattern B) corresponding to the preferred $\mathrm{N}$-terminal proton solvation on the carbonyls at positions (1-2) and (1-3) provides such a test.
Since there is no known fragmentation trend corresponding to this solvation pattern, we examined the whole experimentally available range of $\mathrm{CAD}$ excitations. After lowering the excitation to very small levels while simultaneously extending CAD duration to $400 \mathrm{~ms}$, a new, very distinct fragmentation pathway (Class III) emerged with the C-terminal loss of two amino acids in $\mathrm{A}_{8} \mathrm{R}$ and $\mathrm{A}_{9} \mathrm{R}$ dications (Figure 4). This finding confirmed the MDS prediction provided by pattern $\mathrm{B}$ (Figure 3 ) and, thus, added credibility that the pattern $\mathrm{A}$ is related to Class I fragmentation.

At higher levels and shorter excitation times, the Class III behavior disappeared, being replaced by Class I and II fragmentation (Figure 1). Unlike Class I and III that directly follow from the MDS predictions, Class II behavior is not apparent from the probability maps in Figure 3 for larger
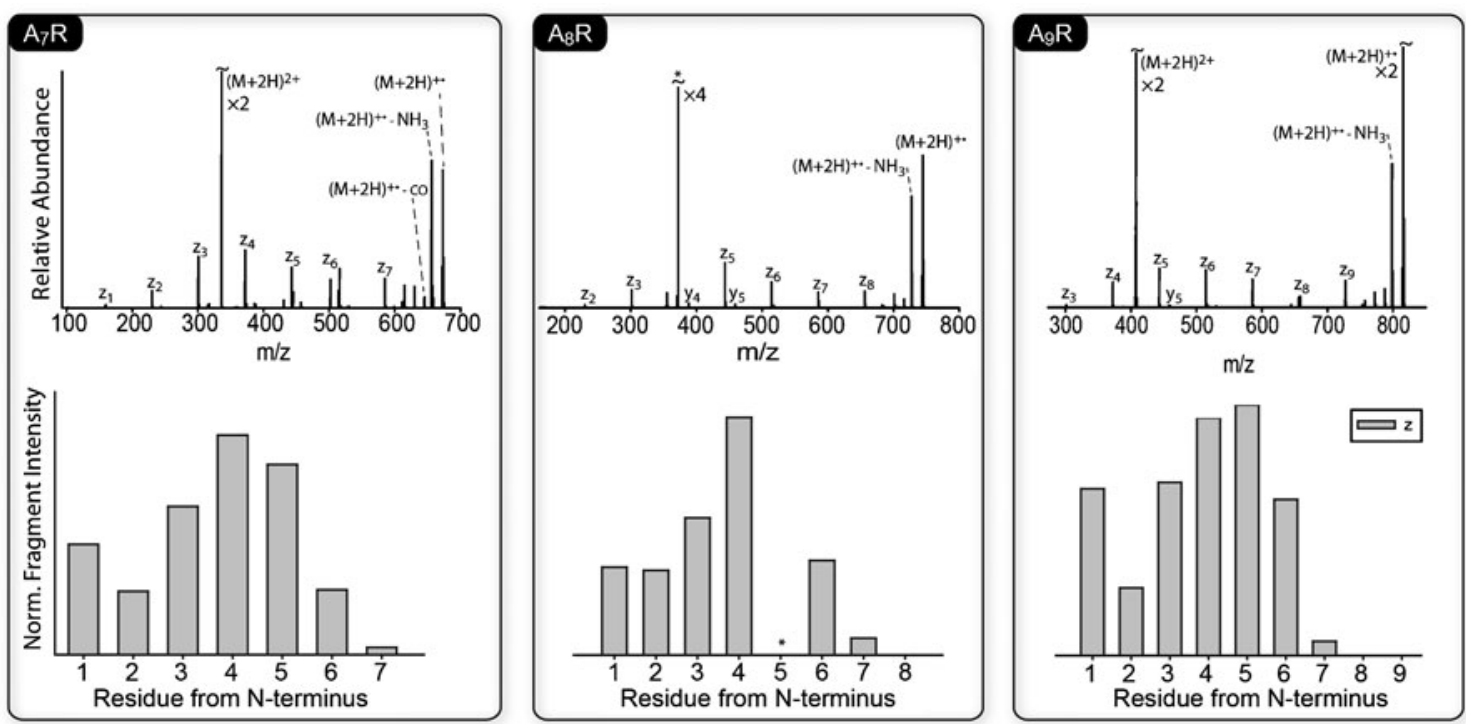

Figure 6. ETD fragmentation of peptide dications with seven, eight, and nine alanine residues; ${ }^{*}$ in $A_{8} R$ indicates $m / z$ of the fragment $z_{4}$ overlaps with precursor 
peptides, and it must arise from the destruction of both $\mathrm{N}$ and C-terminal charge-solvation patterns $\mathrm{A}$ and $\mathrm{B}$ and the onset of proton mobility (vide infra).

Simulating free proton transfer with MDS was beyond the scope of the current study. Here we just notice that due to the lesser basicity of the N-terminal amine compared to Arg guanidinium, the origin of the mobilized proton would conventionally be assigned to the $\mathrm{N}$-terminus. However, mobilization of the Arg proton is also a possibility: CAD (we only have HCD data) of $1^{+}$ions of model peptides gave Class II fragmentation dominated by $\mathrm{b}_{\mathrm{k}}{ }^{+}$ions peaking for at $\mathrm{k}=4-6$ (Figure 5).

Comparison of CAD and ETD results in Figure 6 reveals opposite trends in relation to the cleavage frequency of the second peptide bond. While in CAD this cleavage is enhanced, in ETD it is suppressed. Accepting the above explanation linking the enhanced abundance of $\mathrm{b}_{2}{ }^{+}$ions to the preferred charge solvation on the second carbonyl, we conclude that such solvation negatively correlates with the probability of bond cleavage in ETD. Qualitatively similar results (anti-correlation between charge solvation and ECD cleavage frequencies) have previously been obtained in MDS of a set of 20-residue peptides [26]. Within the framework of the "non-local" ECD/ETD mechanism in which the bond cleavage occurs at a distant site compared with the residing charge [26], this result confirms the MDS-predicted dominance of charge solvation on the second carbonyl.

\section{Conclusions}

Even for homogeneous sequences, fragmentation behavior of tryptic peptide dications is a complex phenomenon that is determined by statistical patterns of charge solvation on and drifting along the backbone carbonyls. The existence of an $\mathrm{N}$-terminal persistent (in a statistical sense) charge-solvation structure was confirmed. This strengthens the model [13] in which this structure, now understood as a charge-solvation pattern, is the origin of Class I fragmentation. The results of the current work once again prove that MDS can correctly predict the proton location distribution and, thus, fragmentation behavior of a peptide. The issue of the exact structure of $\mathrm{b}_{2}{ }^{+}$ions remains outside the scope of this model.

\section{Acknowledgments}

The authors acknowledge support for this work was supported by the Swedish Research Council (grant 20074410 to R.A.Z.). D.M.G. is grateful for a postdoctoral fellowship from the Wenner-Gren Foundation.

\section{References}

1. Mitchell, W.J., McLuckey, S.A.: Collision-induced dissociation (CID) of peptides and proteins. Methods Enzymol. 402, 148-185 (2005)

2. Savitski, M.M., Fälth, M., Fung, Y.M.E., Adams, C.M., Zubarev, R.A. Bifurcating fragmentation behavior of gas-phase tryptic peptide dications in collisional activation. J. Am. Soc. Mass Spectrom. 19, 1755 1763 (2008)
3. Paizs, B., Suhai, S.: Fragmentation pathways of protonated peptides. Mass Spectrom. Rev. 24, 508-548 (2005)

4. Paizs, B., Suhai, S.: Combined quantum chemical and RRKM modeling of the main fragmentation pathways of protonated GGG. I. Cis-trans isomerization around protonated amide bonds. Rapid Commun. Mass Spectrom. 15, 2307-2323 (2001)

5. Cordero, M.M., Houser, J.J., Wesdemiotis, C.: Neutral products formed during backbone fragmentations of protonated peptides in tandem mass spectrometry. Anal. Chem. 65, 1594-1601 (1993)

6. Yalcin, T., Khouw, C., Csizmadia, I.G., Peterson, M.R., Harrison, A.G.: Why are B ions stable species in peptide spectra? J. Am. Soc. Mass Spectrom. 6, 1165-1174 (1995)

7. Yoon, S.H., Chamot-Rooke, J., Perkins, B.R., Hilderbrand, A.E., Poutsma, J.C., Wysocki, V.H.: IRMPD spectroscopy shows that AGG forms an oxazolone b2 ${ }^{+}$Ion. J. Am. Chem. Soc. 130, 17644-17645 (2008)

8. Farrugia, J.M., O'Hair, R.A.J., Reid, G.E.: Do all b2 ions have oxazolone structures? Multistage mass spectrometry and ab initio studies on protonated $\mathrm{N}$-acyl amino acid methyl ester model systems. Int. J. Mass Spectrom. 210/211, 71-87 (2001)

9. Bythell, B.J., Somogyi, A., Paizs, B.: What is the structure of b2 ions generated from doubly protonated tryptic peptides? J. Am. Soc. Mass Spectrom. 20, 618-624 (2009)

10. Bythell, B.J., Erlekam, U., Paizs, B., Maitre, P.: Infrared spectroscopy of fragments from doubly protonated tryptic peptides. Chem. Phys. Chem. 10, 883-885 (2009)

11. Oomens, J., Young, S., Molesworth, S., van Stipdonk, M.: Spectroscopic evidence for an oxazolone structure of the b2 fragment ion from protonated tri-alanine. J. Am. Soc. Mass Spectrom. 20, 334-339 (2009)

12. Perkins, B.R., Chamot-Rooke, J., Yoon, S.H., Gucinski, A.C., Somogyi, A., Wysocki, V.H.: Evidence of diketopiperazine and oxazolone structures for $\mathrm{HA} \mathrm{b}^{+}$Ion. J. Am. Chem. Soc. 131, 1752817529 (2009)

13. Savitski, M.M., Kjeldsen, F., Nielsen, M.L., Zubarev, R.A.: Complementary sequence preferences of electron-capture dissociation and vibrational excitation in fragmentation of polypeptide polycations. Angew. Chem. Int. Ed. 118, 5427-5429 (2006)

14. Tsaprailis, G., Nair, H., Wysocki, V.H., Zhong, W., Futrell, J.J., Summerfield, S.G., Gaskell, S.J.: Influence of secondary structure on the fragmentation of protonated peptides. J. Am. Chem. Soc. 121, 51425154 (1999)

15. Zhang, Z., Bordas-Nagy, J.: Peptide conformation in gas phase probed by collision- induced dissociation and its correlation to conformation in condensed phases. J. Am. Chem. Soc. 17, 786-794 (2006)

16. Savitski, M.M., Kjeldsen, F., Nielsen, M.L., Garbuzynskiy, S.O., Galzitskaya, O.V., Surin, A.K., Zubarev, R.A.: Backbone carbonyl group basicities are related to gas-phase fragmentation of peptides and protein folding. Angew. Chem. Int. Ed. 46, 1481-1484 (2007)

17. Burlet, O., Yang, C.Y., Gaskell, S.: Influence of cysteine to cysteic acid oxidation on the collision-activated decomposition of protonated peptides: Evidence for intraionic interactions. J. Am. Soc. Mass Spectrom. 3, 337-344 (1992)

18. Dongré, A.R., Jones, J.L., Somogyi, Á., Wysocki, V.H.: Influence of peptide composition, gas-phase basicity, and chemical modification on fragmentation efficiency: evidence for the mobile proton model. J. Am. Chem. Soc. 118, 8365-8374 (1996)

19. Harrison, A.G., Yalcin, T.: Proton mobility in protonated amino acids and peptides. Int. J. Mass Spectrom. 165/166, 339-347 (1997)

20. Harrison, A.G.: Charge-separation reactions of doubly-protonated peptides: Effect of peptide chain length. J. Am. Soc. Mass Spectrom. 20, 1890-1895 (2009)

21. Harrison, A.: Effect of the identity of Xaa on the fragmentation modes of doubly-protonated Ala-Ala-Xaa-Ala-Ala-Ala-Arg. J. Am. Soc. Mass Spectrom. 22, 906-911 (2011)

22. Samuelson, S., Martyna, G.J.: Computer simulation studies of finite temperature conformational equilibrium in alanine-based peptides. $J$. Phys. Chem. B. 103, 1752-1766 (1999)

23. Olsen, J.V., Schwartz, J.C., Griep-Raming, J., Nielsen, M.L., Damoc, E., Denisov, E., Lange, O., Remes, P., Taylor, D., Splendore, M., Wouters, E.R., Senko, M., Makarov, A., Mann, M., Horning, S.: A Dual Pressure Linear Ion Trap Orbitrap Instrument with Very High Sequencing Speed. Mol. Cell. Prot. 8, 2759-2769 (2009)

24. Syka, J.E.P., Coon, J.J., Schroeder, M.J., Shabanowitz, J., Hunt, D.F.: Peptide and protein sequence analysis by electron transfer dissociation mass spectrometry. Proc. Natl. Acad. Sci. U.S.A. 101, 9528-9533 (2004) 
25. McAlister, G.C., Phanstiel, D., Good, D.M., Berggren, W.T., Coon, J.J.: Implementation of electron-transfer dissociation on a hybrid linear ion trap-orbitrap mass spectrometer. Anal. Chem. 79, 3525-3534 (2007)

26. Patriksson, A., Adams, C., Kjeldsen, F., Raber, J., van der Spoel, D., Zubarev, R.A.: Prediction of $\mathrm{NC} \alpha$ bond cleavage frequencies in electron capture dissociation of Trp-cage dications by force-field molecular dynamics simulations. Int. J. Mass Spectrom. 248, 124-135 (2006)

27. Adams, C.M., Kjeldsen, F., Patriksson, A., van der Spoel, D., Gräslund, A., Papadopoulos, E., Zubarev, R.A.: Probing solution- and gas-phase structures of Trp-cage cations by chiral substitution and spectroscopic techniques. Int. J. Mass Spectrom. 253, 263-273 (2006)

28. Patriksson, A., Adams, C.M., Kjeldsen, F., Zubarev, R.A., van der Spoel, D.: A direct comparison of protein structure in the gas and solution phase: The Trp-cage. J. Phys. Chem. B 111, 13147-13150 (2007)

29. Van der Spoel, D., Lindahl, E., Hess, B., Groenhof, G., Mark, A.E., Berendsen, H.J.C.: GROMACS: Fast, flexible, and free. J. Comp. Chem. 26, 1701-1709 (2005)

30. Hess, B., Kutzner, C., van der Spoel, D., Lindahl, E.: GROMACS 4: Algorithms for highly efficient, load-balanced, and scalable molecular simulation. J. Chem. Theory Comput. 4, 435-447 (2008)

31. Jorgensen, W.L., Maxwell, D.S., Tirado-Rives, J.: Development and testing of the OPLS all-atom force field on conformational energetics and properties of organic liquids. J. Am. Chem. Soc. 118, 11225-11236 (1996)
32. Jorgensen, W.L., Tirado-Rives, J.: Potential energy functions for atomic-level simulations of water and organic and biomolecular systems. Proc. Natl. Acad. Sci. U. S. A. 102, 6665-6670 (2005)

33. Hukushima, K., Nemoto, K.: Exchange Monte Carlo method and application to spin glass simulations. J. Phys. Soc. Jpn. 65, 1604-1608 (1996)

34. Patriksson, A., van der Spoel, D.: A temperature predictor for parallel tempering simulations. Phys. Chem. Chem. Phys. 10, 20732077 (2008)

35. Goloborodko, A., Gorshkov, M., Good, D., Zubarev, R.: Sequence scrambling in shotgun proteomics is negligible. J. Am. Soc. Mass Spectrom. 22, 1121-1124 (2011)

36. McCormack, A.L., Somogyi, A., Dongre, A.R., Wysocki, V.H.: Fragmentation of protonated peptides: surface-induced dissociation in conjunction with a quantum mechanical approach. Anal. Chem. 65, 2859-2872 (1993)

37. Tang, X.J., Thibault, P., Boyd, R.K.: Fragmentation reactions of multiply-protonated peptides and implications for sequencing by tandem mass spectrometry with low-energy collision-induced dissociation. Anal. Chem. 65, 2824-2834 (1993)

38. Vachet, R.W., Bishop, B.M., Erickson, B.W., Glish, G.L.: Novel peptide dissociation: Gas-phase intramolecular rearrangement of internal amino acid residues. JACS 119, 5481-5486 (1997) 